JOURNAL OF RESEARCH of the National Bureau of Standards-B. Mathematical Sciences

Vol. $80 \mathrm{~B}, \mathrm{No} .3$, July-September 1976

\title{
A Note on Pairs of Matrices With Product Zero
}

\section{Charles R. Johnson*}

\author{
Institute for Basic Standards, National Bureau of Standards, Washington, D.C. 20234 \\ May 20, 1976
}

\begin{abstract}
Pairs of not necessarily hermitian matrices for which $A B=B A=0$ are characterized in terms of the singular values of $A+B$. This provides a generalization and a simpler proof of a classical result on the independence of quadratic forms in normal random variables.
\end{abstract}

Key words: Eigenvalues; independence; quadratic form; random variable; singular value decomposition.

Suppose that

$$
x^{T}=\left(x_{1}, \ldots, x_{n}\right)
$$

is a vector of independent normal 0,1 random variables. In [3] ${ }^{1}$ it is pointed out that

$$
y_{1}=x^{T} A x \text { and } y_{2}=x^{T} B x
$$

are independent random variables, where $A$ and $B$ are real symmetric matrices, if and only if $A B=0$. An explanation of this fundamental fact is given in [5] and amounts to a somewhat lengthy derivation of the following:

Theorem I: If $\mathrm{A}$ and $\mathrm{B}$ are real symmetric matrices with eigenvalues $\left\{\lambda_{1}, \ldots, \lambda_{r}, 0, \ldots, 0\right\}$ and $\left\{\lambda_{r+1}, \ldots, \lambda_{n}, 0, \ldots, 0\right\}$ respectively, then $\mathrm{A}+\mathrm{B}$ has eigenvalues $\left\{\lambda_{1}, \ldots, \lambda_{n}\right\}$ if and only if $\mathrm{AB}=0$.

The independence of $y_{1}$ and $y_{2}$ is, in a straightforward way, equivalent to $A+B$ having eigenvalues $\lambda_{1}$. . ., $\lambda_{n}$, and Theorem I (which was first noted by Craig [3]) is sufficiently fundamental that generally it is now at least stated in advanced texts. For example, a portion of a proof is given in [4]. Apparently in ignorance of [3, 4,5], an alternate proof of Theorem I is given in [1].

Our goal is to give a generalization of Theorem I whose proof is quite simple. In addition to including a rather different proof of Theorem I, our observation points out that the symmetry of $A$ and $B$ is not an essential assumption. We recall that the singular values of a general complex matrix $A$ are, by definition, the nonnegative square roots of the eigenvalues of $A^{*} A$. A good general reference on the singular values decomposition of a matrix is [6].

Theorem II: Suppose A and B are n-by-n complex matrices with singular values $\left\{\mathrm{d}_{1}, . . ., \mathrm{d}_{r}, 0\right.$, ..., 0$\}$ and $\left\{\mathrm{d}_{r+1}, \ldots, \mathrm{d}_{n}, 0, \ldots, 0\right\}$ respectively. Then $A+B$ has singular values $\left\{\mathrm{d}_{1}, \ldots\right.$, $\left.\mathrm{d}_{n}\right\}$ if and only if $\mathrm{AB}=\mathrm{BA}=0$.

Proof: We assume, without loss of generality, that $d_{1}, \ldots, d_{n}$ are nonzero and that

$$
A=\left(\begin{array}{ll}
D_{1} & 0 \\
0 & 0
\end{array}\right),
$$

where

$$
D_{1}=\operatorname{diag}\left\{d_{1}, \ldots, d_{r}\right\} \text {. }
$$

AMS Subject Classification: 15 A24, 60-E05, 62-H99.

${ }^{*}$ Present address: Institute for Physical Science and Technology, Univarsity of Maryland, College Park, Md. 20742.

1 Figures in brackets indicate literature references at the end of this paper. 


$$
B=U^{*}\left(\begin{array}{ll}
0 & 0 \\
0 & D_{2}
\end{array}\right) V
$$

be a singular value decomposition of $B$, where

$$
D_{2}=\operatorname{diag}\left\{d_{\tau+1}, \ldots, d_{n}\right\}
$$

and where the unitary matrices $U$ and $V$ are partitioned

$$
U=\left(\begin{array}{ll}
U_{1} & U_{2} \\
U_{3} & U_{4}
\end{array}\right), \quad V=\left(\begin{array}{ll}
V_{1} & V_{2} \\
V_{3} & V_{4}
\end{array}\right)
$$

conformally with $D_{1}$ and $D_{2}$. First, suppose that $A+B$ has singular values $\left\{d_{1}, \ldots, d_{n}\right\}$. We then have

$$
\begin{aligned}
& d_{1} d_{2} \ldots d_{n}=|\operatorname{det}(A+B)| \\
= & \operatorname{det}\left[\left(\begin{array}{ll}
D_{1} & 0 \\
0 & 0
\end{array}\right)+U^{*}\left(\begin{array}{ll}
0 & 0 \\
0 & D_{2}
\end{array}\right) V\right] \\
= & \operatorname{det}\left[U\left(\begin{array}{ll}
D_{1} & 0 \\
0 & 0
\end{array}\right)+\left(\begin{array}{ll}
0 & 0 \\
0 & D_{2}
\end{array}\right) V\right] \\
= & \operatorname{det}\left[\left(\begin{array}{ll}
U_{1} D_{1} & 0 \\
U_{3} D_{1} & 0
\end{array}\right)+\left(\begin{array}{ll}
0 & 0 \\
D_{2} V_{3} & D_{2} V_{4}
\end{array}\right)\right] \\
=\left|\operatorname{det} U_{1} D_{1} \operatorname{det} D_{2} V_{4}\right|=d_{1} d_{2} \ldots . & d_{n}\left|\operatorname{det} U_{1} \operatorname{det} V_{4}\right| .
\end{aligned}
$$

This implies that $\mid \operatorname{det} U_{1}$ det $V_{4} \mid=1$ which, because of Hadamard's determinantal inequality, in turn implies that $U_{1}$ and $V_{4}$ are both unitary. This means that $U_{2}, U_{3}, V_{2}$ and $V_{3}$ are all 0 , that

$$
B=\left(\begin{array}{cccc}
0 & & 0 & \\
0 & U^{*}{ }_{4} & D_{2} & V_{4}
\end{array}\right),
$$

and that $A B=B A=0$.

On the other hand, suppose that $A B=B A=0$. If $B$ is partitioned,

$$
B=\left(\begin{array}{ll}
B_{1} & B_{2} \\
B_{3} & B_{4}
\end{array}\right),
$$

as $U$ and $V$, then $A B=0$ implies that $B_{1}=0$ and $B_{2}=0$, while $B A=0$ implies that $B_{1}=0$ and $B_{3}=0$. Thus, $B_{4}$ has singular values $\left\{d_{r+1}, \ldots, d_{n}\right\}$, and

$$
A+B=\left(\begin{array}{ll}
D_{1} & 0 \\
0 & B_{4}
\end{array}\right)
$$

has singular values $\left\{d_{1}, \ldots, d_{n}\right\}$ as was to be shown.

Of course, for hermitian matrices $A B=0$ is equivalent to $B A=0$. However, for nonhermitian matrices, $A B=0$ does not mean that $B A=0$ so that the condition $A B=B A=0$ may not be weakened in theorem II. It should, however, be noted that, as can be easily seen via the proof of theorem II, the assumption $A B=B A=0$ implies $\left(A+A^{*}\right)\left(B+B^{*}\right)=0$. 


\section{References}

[1] Aitken, A. C. On the statistical independence of quadratic forms in normal variates, Biometrika 37, 93-96 (1950).

[2] Brand, L. On the product of singular symmetric matrices, Proc. AMS 22, 377 (1969).

[3] Craig, A. T. Note on the independence of certain quadratic forms, Annals of Math. Stat. 14, 195-197 (1943).

[4] Graybill, F. A. An Introduction to Linear Statistical Models (McGraw-Hill, New York, 1961).

[5] Hotelling, H. Note on a matric theorem of A. T. Craig, Annals of Math. Stat. 15, 427-429 (1944).

[6] Stewart, G. W. Introduction to Matrix Computations (Academic Press, New York, 1973).

(Paper 80B3-447) 